\title{
La Competencia Digital Docente, una revisión sistemática de los modelos más utilizados
}

\section{The Digital Teaching Competence, a systematic review of the most commonly used models}

\author{
David Jiménez-Hernández \\ Universidad Miguel Hernández (España) \\ djimenez@umh.es \\ Práxedes Muñoz Sánchez \\ Universidad Católica de Murcia (España) \\ pmunoz@ucam.edu \\ Fulgencio S. Sánchez Giménez \\ IES Jaume Primer Ontinyent (España) \\ fsanchez@iesjaumeprimer.net
}

Recibido: 10/03/2021

Aceptado: $22 / 03 / 2021$

Publicado: 01/06/2021

\section{RESUMEN}

Partiendo del interés social y educativo actual, este artículo pretende conocer el estado de la cuestión en el campo de la Competencia Digital Docente (CDD), con la finalidad de saber cómo se encuentra el trabajo de estas destrezas tanto en el ámbito teórico como empírico. Para ello se han recopilado diferentes modelos que destacan por su uso como guías de formación para facilitar su desarrollo. Este estudio ha tenido en cuenta diferentes países e instituciones, así como sus características, finalidades y dimensiones. Además, en todas se remarcan investigaciones que los modelos emplean para favorecer la adquisición de estas competencias, así como su análisis en controversias y perspectivas de la comunidad educativa y la praxis en las políticas educativas. A lo largo de todos estos modelos de CDD, se ha podido comprobar la gran carga de responsabilidad tanto a nivel institucional como del profesorado en la adquisición de competencias digitales, para poder responder a las demandas sociales y educativas planteadas en todos los niveles de formación.

\section{PALABRAS CLAVE}

Competencia digital; personal docente; construcción de modelos; investigación educativa; TIC.

\section{ABSTRACT}

Due to the current interest in the Teacher Digital Competence (TDC), both from the social and education fields, this article shows its state-of-art, in order to find out how the work on these skills is going both theoretically and empirically. Thus, we compile different models that stand out as training guides that facilitate TDC's development. We analyze different countries and institutions for this study, as well as their characteristics and purposes. For each model, we highlight research results that the models are based on to facilitate gaining TDC skills. We analyze how the educational community evaluates them and their results after their implementation in educational policy. We reveal that each TDC model implies a great burden of responsibility for institutions and educators. In order to be able to respond to the social and educational requirements raised at all levels of training, all models strongly rely on the educational community to gain digital competence. 


\section{KEYWORDS}

Digital competence; teaching personal; model construction; educational research; ICT.

\section{CITA RECOMENDADA}

Jiménez-Hernández, D., Muñoz, P. \& Sánchez, F.S. (2021). La Competencia Digital Docente, una revisión sistemática de los modelos más utilizados. Revista Interuniversitaria de Investigación en Tecnología Educativa, 10, 105-120. https://doi.org/10.6018/riite.472351

\section{Principales aportaciones del artículo y futuras líneas de investigación:}

- Recoger en un artículo los principales modelos de la Competencia Digital Docente (CDD) empleados en diferentes sistemas educativos.

- Identificar algunas de las investigaciones que se han desarrollado a partir de los modelos de CDD.

- Establecer las bases teóricas de futuros estudios por medio de la creación un instrumento de recogida de información y análisis de CDD.

\section{INTRODUCCIÓN}

En la sociedad actual se exige a las nuevas generaciones un nivel de competencia digital que va más allá del uso recreativo de dispositivos, razón por la cual, es necesario que desde los centros educativos puedan aprender cómo utilizarlos de manera didáctica y segura. Para conseguirlo, se requiere de un profesorado que esté cualificado para abordar el proceso de enseñanza-aprendizaje de los discentes con garantías y, es aquí, donde radica la importancia de conocer más a fondo qué es la Competencia Digital Docente (CDD), y algunos de los diferentes modelos que se han desarrollado para su formación. Tal y como lo expone el Ministerio de Educación y Formación Profesional (2020) en el marco de referencia de la CDD, es necesario que el profesorado y los centros educativos posean un adecuado grado de competencia digital, que favorezca la adquisición de competencias clave en el alumnado y en los ciudadanos.

Antes de entrar a valorar la CDD, se comenzará especificando qué es la Competencia digital. Según la Ley Orgánica de la Mejora de la Calidad Educativa (LOMCE, 2013, p.10), la CDD se define como:

Un conjunto de destrezas para investigar y procesar información en nuevos productos de conocimiento. Y para ello se debe de conseguir un conocimiento básico de aplicaciones informativas, adaptándolas al conocimiento y a la resolución de problemas basados en contexto reales, y adquiriendo un pensamiento crítico de las fuentes y canales de información provenientes de las Tecnologías de la Información y la Comunicación (TIC).

La competencia digital debe de ser transversal, que se trabaje en todas las áreas del currículum, lleve a un perfeccionamiento continuo y constante que aumente el rendimiento académico y personal (Guillén-Gámez et al., 2018).

Para llegar a este punto, es necesario que todos los docentes posean una competencia digital que permita la transmisión de los conocimientos y destrezas mencionadas a todo el alumnado. Por esta razón, según Esteve (2015), resulta difícil concebir que el profesorado no tenga una competencia digital mínima, ya que no estará preparando a sus estudiantes para los retos sociales y educativos que propone la sociedad en la actualidad. Por ello, será clave que el docente sepa crear y ofrecer contenidos digitales a su alumnado en diferentes situaciones y contextos.

A partir de aquí habría que responder a la pregunta; ¿qué se entiende por CDD? Son aquellas herramientas, actitudes y conocimientos referentes a ámbitos tecnológicos, comunicativos, informacional y mediático, que dan lugar a la alfabetización docente y que les sirve tanto a nivel personal como para garantizar una educación de calidad a su alumnado (Gisbert \& Esteve, 2016). Posteriormente, Lázaro \& Gisbert (2015) consideraron que un buen 
nivel de CCD, favorece el empleo eficaz de medios TIC y su adaptación a las necesidades del alumnado. Además, la importancia de la formación de los docentes en ésta, radica en la respuesta que desde el sistema educativo se debe dar al avance social y al mercado laboral. Según Durán et al. (2019), la CCD está compuesta por: las dimensiones propias de la competencia digital, con componentes tecnológicos, informacionales y de alfabetización multimedia; y por el empleo de diferentes tecnologías en sus aulas con criterios pedagógicos.

En este sentido, Krumsvik (2011) considera un factor clave para que el alumnado pueda adquirir una concepción amplia de la competencia digital, que el profesorado también disponga de ésta. A medida que el profesorado mejore esta competencia, irá utilizando más las TIC en ambientes escolares, hecho este que le inducirá a realizar más prácticas educativas relevantes, e incluso, a poder innovar con las experiencias didácticas que permitan mejores resultados de aprendizaje. No obstante, y como remarca el autor, es también vital que todo docente utilice criterios pedagógicos además de los tecnológicos para la selección y utilización de recursos TIC con los discentes, así se podrá extraer todo su potencial.

Por todos los argumentos expresados, se vio necesario realizar un trabajo de esta índole en el que se aglutinen los principales modelos de CDD que se han ido desarrollando en diferentes sistemas educativos, sus características particulares y algunas de las investigaciones que se hayan efectuado a partir de éstas.

Las dimensiones de la CDD desarrolladas en la investigación de Falcó (2017), partieron de las cinco iniciadas por el Instituto Nacional de Tecnologías Educativas y Formación del Profesorado (2013), a la que añade una más, quedando de la siguiente manera:

1. Información y alfabetización informacional. Incluyendo su localización, recuperación, organización, así como la evaluación de la información digital.

2. Comunicación y elaboración. Siendo todo lo referido a expresarse por medio de entornos digitales, además de cooperar y participar empleando herramientas digitales.

3. Crear contenidos digitales. Además de permitir su edición en diferentes formatos, así como contenidos multimedia.

4. Seguridad. Realizando una protección personal, de datos y realizando un uso seguro y sostenible.

5. Resolver problemas. Siendo capaz de elegir la herramienta digital necesaria para una determinada tarea, además de resolver problemas técnicos y digitales.

6. Emplear materiales digitales con fines educativos. Conocimiento y empleo de diferentes soportes digitales creados expresamente o no, que favorezcan la consecución de objetivos con perspectiva crítica.

Una vez que se ha presentado qué es y las principales dimensiones de la CDD, se procederá a explicar el método seguido en el presente estudio.

\section{MÉTODO}

La revisión sistemática de literatura (RSL) se fundamenta en una metodología cualitativa descriptiva con un enfoque de análisis interpretativo crítico (Rodrigo, 2001), y además, desde una perspectiva emic y etic (Pike, 1967), que parte de especialistas en el campo de las nuevas tecnologías y la formación docente, que desde la propia experiencia (Muñoz, 2016) favorece ambas perspectivas, y junto al análisis de contenido, permite una comparación entre los modelos estudiados de una forma más integral.

Según Aguirre \& Jaramillo (2015) el uso de la descripción se fundamenta en tres aspectos: en primer lugar, un diseño descriptivo que perdura en el tiempo y es necesario en el ámbito metodológico, en segundo lugar, protege el encuentro con la diversidad, en este caso con modelos diversos de competencia digital que nos acerca a modos de entender las prioridades, y por último, la descripción es "garante de validez" en el registro y análisis de datos. 
El proceso de investigación está ligado a la exploración de estudios que destacan por el desarrollo y el empeño de abarcar modelos y otras perspectivas sobre las CDD. Por ello, este artículo se ha basado en una búsqueda de bibliografía sobre la citada temática, mediante la exploración en diferentes bases de datos tanto de revistas como de documentación, en: Google Schoolar, Dialnet, Redalyc, Redined y Taylor \& Francis online. El análisis ha sido realizado fuera de limitantes regionales, pero como se puede evidenciar, la información destaca de Europa y América en los idiomas inglés y castellano.

En una revisión sistemática se distinguen tres fases principales: planificación, realización e informe de la revisión. Durante este proceso, existen criterios de inclusión y exclusión, que pueden ser considerados en diferentes momentos del desarrollo de la revisión (Kitchenham, 2004). En esta investigación un de criterio de exclusión empleado ha sido la "formación online", por la dificultad de distinguir al docente online del presencial en una literatura que, a veces, hace complicada esta distinción. En cambio, un criterio de inclusión al que se recurrió fue "metodologías e instrumentos en relación a la alfabetización digital".

El proceso de revisión de literatura comienza en la selección de bases de datos académicas que facilitan, mediante palabras claves, la adquisición de fuentes. Tras ello, se seleccionaron las categorías de análisis realizadas a partir de una primera investigación sobre las CDD, destacando la información en cuanto a: la implantación de los modelos, las revisiones teóricas que ya se han llevado a cabo y las investigaciones y evaluaciones realizadas. La selección se realizó a partir de 786 referencias, siendo las temáticas generales sobre tres categorías: competencia digital, investigaciones y proyectos de intervención y/o innovación. Los criterios de inclusión se basan en las categorías de los modelos de CDD, excluyendo información de tipo general sobre formación digital. Los documentos escogidos finalmente fueron 46 (Tabla 1).

Tabla 1. Selección de literatura sobre Modelos de Competencia Digital Docente.

\begin{tabular}{|c|c|c|c|}
\hline $\begin{array}{c}\text { Base de } \\
\text { datos }\end{array}$ & $\begin{array}{l}\text { Protocolo de } \\
\text { selección }\end{array}$ & Criterios de inclusión & Selección/utilizados \\
\hline Taylor \& & Literatura inglés & Modelos Competencia Digital & $79 / 5$ \\
\hline $\begin{array}{l}\text { Francis } \\
\text { online }\end{array}$ & $\begin{array}{l}\text { y español 2000- } \\
2021\end{array}$ & $\begin{array}{c}\text { Docente y "teacher digital } \\
\text { competence" }\end{array}$ & $6 / 0$ \\
\hline \multirow{5}{*}{$\begin{array}{l}\text { Google } \\
\text { schoolar }\end{array}$} & Literatura inglés & "Modelo de competencia digital & $34 / 15$ \\
\hline & $\begin{array}{c}\text { y español 2000- } \\
2021 \text { y }\end{array}$ & $\begin{array}{l}\text { docente" y artículos } \\
\text { relacionados }\end{array}$ & $11 / 5$ \\
\hline & artículos & & \\
\hline & relacionados & & \\
\hline & & & $15 / 8$ \\
\hline Dialnet & $\begin{array}{l}\text { Literatura inglés } \\
\text { y español 2000- } \\
2021\end{array}$ & $\begin{array}{l}\text { Modelos de Competencia } \\
\text { Digital Docente }\end{array}$ & $309 / 10$ \\
\hline Redalyc & $\begin{array}{l}\text { Literatura inglés } \\
\text { y español 2000- } \\
2021\end{array}$ & CDD & $90 / 1$ \\
\hline Redined & $\begin{array}{l}\text { Literatura inglés } \\
\text { y español 2000- } \\
2021\end{array}$ & CDD/Innovaciones & $237 / 2$ \\
\hline
\end{tabular}

A continuación, se destacan las subcategorías de análisis, que están íntimamente ligadas en la dirección de investigar, a la vez que de fomentar, reaccionar y transformar buenas prácticas en base a la adquisición de competencias digitales.

Tanto las palabras claves de la investigación como las categorías más específicas, han sido analizadas en buscadores de bibliografía académica. Este resumen puede verse en la Tabla 2. 
Tabla 2. Categorías de la revisión bibliográfica en base a CDD

\begin{tabular}{|c|c|c|}
\hline Categorías & Investigaciones & Proyectos/intervención/innovación \\
\hline $\begin{array}{l}\text { Sociedad del } \\
\text { conocimiento }\end{array}$ & Teórica y crítica, RSL & \\
\hline Brecha digital & $\begin{array}{c}\text { RSL, explorativas, } \\
\text { descriptivas y comparativas }\end{array}$ & Formación online \\
\hline $\begin{array}{c}\text { Alfabetización } \\
\text { digital e } \\
\text { instrumentos }\end{array}$ & $\begin{array}{c}\text { RSL, Explorativas, } \\
\text { descriptivas y comparativas }\end{array}$ & \\
\hline $\begin{array}{l}\text { Competencias } \\
\text { digitales }\end{array}$ & $\begin{array}{c}\text { RSL, Explorativas, } \\
\text { descriptivas y comparativas }\end{array}$ & \\
\hline $\begin{array}{l}\text { Modelos de } \\
\qquad C D D\end{array}$ & $\begin{array}{c}\text { RSL, Investigaciones } \\
\text { cualitativas y cuantitativas }\end{array}$ & Puesta en prácticas CDD \\
\hline $\begin{array}{l}\text { Formación y } \\
\text { formadores } \\
\text { en } C D D\end{array}$ & $\begin{array}{l}\text { Investigaciones cualitativas } \\
\text { y cuantitativas }\end{array}$ & Perfeccionar CDD \\
\hline Evaluaciones & $\begin{array}{l}\text { RSL, Investigaciones } \\
\text { cuantitativas Instrumentos } \\
\text { (cuestionarios validados) }\end{array}$ & $\begin{array}{c}\text { Proyectos y elaboración de instrumentos de } \\
\text { evaluación }\end{array}$ \\
\hline
\end{tabular}

En base a toda la información aportada, el objetivo general del artículo será realizar un estudio sobre algunos de los principales modelos de CDD desarrollados hasta el momento, que permita clarificar y conocer el estado de la cuestión sobre esta temática, comprobando sus principales características, campos de aplicación, formaciones e investigaciones derivadas de ésta.

\section{MODELOS DE ESTUDIO DE LA COMPETENCIA DIGITAL DOCENTE}

\subsection{ECD-TIC}

El primer modelo que se analiza es el denominado, Estándares de Competencias TIC (Tecnología de la Información y la Comunicación) para Docentes (ECD-TIC) desarrollado por UNESCO (2008). A partir de este, se ofrecen diferentes formaciones que capaciten al profesorado para el empleo de TIC y para desarrollar innovaciones pedagógicas, que dé lugar a clases más dinámicas y al uso de diferentes métodos didácticos (Girón et al., 2019). De esta manera se crea un marco en el que se desarrollarán estándares en competencias TIC, que fueran destinados tanto para los docentes ejercientes como para el desarrollo de programas de formación inicial (Esteve, 2015).

Este modelo pone el énfasis en integrar las TIC: en la pedagogía, en la formación de los docentes, en los currículums, en las políticas educativas y en los sistemas de gestión escolar. Por lo cual, pretende que se mejore no sólo las prácticas docentes, sino que sirva como modelos de gestión de calidad del sistema de educación de un país, y que al final, eso incida en su desarrollo económico y social (UNESCO, 2008). Según Esteve (2015), este modelo desarrolló un marco en el que se pretendía: que las diferentes instituciones que realizan formación a los docentes, tengan una guía para realizar programas formativos y desarrollar material de aprendizaje; que los docentes tengan cualificaciones para integrar las TIC en el aula; y establecer unas pautas generales sobre las diferentes ideas y el vocabulario de las TIC en educación. 
De acuerdo con Ballesta et al. (2016), la UNESCO (2008) persigue el desarrollo de un sistema educativo de la siguiente manera:

a) Un centro educativo en el que el currículo empleado sea prescriptivo, con un rol de docente activo y el trabajo del alumnado en pequeños grupos o individual y presenciales.

b) El modelo de enseñanza seguido es el cognitivo-constructivista.

c) Entorno de aprendizaje presencial utilizando TIC.

d) Uso de herramientas digitales, centrando el proceso de enseñanza-aprendizaje en la tecnología, a través de Internet y software de comunicación, gestión y presentación multimedia.

Finalmente, la UNESCO en su versión de 2011, especificó tres factores básicos: alfabetización tecnológica, profundización y creación de conocimientos. De estos se desarrollaron seis aspectos clave que fueron: plan de estudio y evaluación, pedagogía, TIC, formación docente y organización y administración. A partir de aquí se crea un marco con 18 módulos formativos.

Un ejemplo de investigación en la que se desarrolló el modelo de la UNESCO (2008), fue para crear un instrumento que permitiera medir el grado de competencias digitales del profesorado en educación básica (Valdivieso \& Gonzáles, 2016).

\subsection{NETS-T}

Es un sistema empleado en EE. UU, en el que la International Society for Technology in Education (ISTE, 2008) desarrolló los National Educational Technology Standards for Teachers (NETS-T). A partir del modelo, diferentes estados del país han elaborado sus propios estándares y manuales para ayudar a la implementación de tecnologías en las aulas, partiendo de una concepción educativa y de las TIC: holista, transdisciplinar y próximo al constructivismo (Morphew, 2012). Como tal, se centra en la manera en que los docentes mejoran el aprendizaje de sus estudiantes utilizando TIC, partiendo del empleo didáctico de éstas para el diseño y evaluación de actividades o materiales, pero también contemplan otros aspectos como la formación del docente o la ciudadanía digital (Castañeda et al., 2018).

El profesorado digitalmente competente es aquel que sirve a la comunidad educativa con buenas prácticas de acuerdo con los cinco ejes o dimensiones (ISTE, 2008):

a) Facilitar el aprendizaje y los procesos creativos de su alumnado, usando las TIC para solucionar problemas acontecidos en su entorno real, favorecer el trabajo cooperativo, creación de nuevos conocimientos, etc.

b) Diseñar prácticas de aprendizaje relevantes, mediante la búsqueda de experiencias enriquecedoras de recursos web que fomenten la creatividad, la curiosidad o la diversidad.

c) Ofrecer herramientas digitales útiles para la formación autónoma y el aumento de la productividad académica.

d) Promover el constructo del e-ciudadano, dando a conocer un uso seguro y ético de las TIC.

e) Comprometerse con el crecimiento profesional y el liderazgo introduciendo recursos digitales.

Para cada uno de estos niveles de competencia, se establece posteriormente varios grados de desempeño, que van desde el principiante, propio del profesorado en formación que se acerca por primera vez a las TIC, pasando por medio y experto, hasta el transformador, a los que llegan quienes son capaces de desarrollar estrategias que son pioneras en el ámbito de la innovación educativa. 
Este modelo ha sido utilizado en investigaciones para conocer el nivel de competencia digital del profesorado en activo y en formación (Banister \& Vannatta, 2012), y también solamente en los futuros docentes (Esteve et al., 2016a; Esteve et al., 2016b; Kumar \& Vigil, 2014).

Dentro de este apartado, también se desarrollará el modelo ISTE II (2017) como una revisión y ampliación del modelo ISTE I (2007) que, realizando una serie de modificaciones, recoge un modelo donde, por un lado, se enfatiza en la capacitación digital docente, la actualización de ésta y el hecho de compartir las buenas prácticas relacionadas con las TIC, no solo entre su alumnado, sino también con el resto de profesorado de su entorno presencial y virtual. Además, por otro lado, confiere más autonomía al alumnado para que sean sujetos activos de su proceso de enseñanza-aprendizaje. En definitiva, de forma holística y transdisciplinar (Biel \& Álvarez, 2019). En su actualización más reciente (ISTE, 2017), cambia la perspectiva y la dirige más hacia los estudiantes, para que desarrollen de manera más autónoma sus aprendizajes. Como en la anterior, proponen perfiles y habilidades a los docentes para que sean capaces de realizar este nuevo rol.

De esta manera, se fomenta una metodología más activa del proceso de enseñanzaaprendizaje donde docentes y alumnado colaboran por conseguir unas metas comunes. El modelo enfatiza siete dimensiones que el profesorado debe trabajar y evaluar en su práctica educativa (ISTE, 2017): 1. Formadores en constante formación; 2. Líderes en el uso de las TIC; 3. Ciudadanos como modelos de ejemplos; 4. Incentivador del aprendizaje; 5. Diseñador de experiencias de aprendizaje individualizadas; 6. Fomentadores del trabajo autónomo del alumnado; y 7 . Analistas de los procesos evaluativos y comunicativos mediante el uso de las TIC. A la vez, esta propuesta está abierta a la investigación, a compartir buenas prácticas y temas e ideas influyentes, pero también las notificaciones que pueden causar problemas como datos falsos (Torrey, 2018).

\subsection{ENLACES}

Del modelo NETS-T se derivaron programas como ENLACES, desarrollado por el Ministerio de Educación de Chile (2006), con un supuesto análisis histórico desde la intención de volcar sus políticas educativas en lograr recursos y competencias en todos los centros educativos y favorecer valores en cuanto a desarrollo humano, justicia y democracia (Bilbao \& Salinas, 2010). En el año 2007 el Ministerio de Educación de Chile, a través del Centro de Educación y Tecnología, publicó un informe con el que pretende definir la competencia TIC para la profesión docente y establecer los estándares para los programas de formación en esta temática, documento que fue actualizado en 2011 (Enlaces, 2011). Esteve (2015) destaca en este modelo dos habilidades: en recursos y en conocimientos. Por ello recoge cómo los docentes deben trabajar las TIC en diferentes áreas, y no únicamente centradas en la consecución de requerimientos pedagógicos en el aula. Se desarrolla en cinco dimensiones: pedagógica; técnica (o instrumental); de gestión, desarrollo o fortalecimientos de procesos de aprendizajes; social, ética y legal; y de desarrollo y responsabilidad profesional (Biel \& Álvarez, 2019). De estas dimensiones se establecen las competencias: comunicación, innovación, capacidad de planificar y organizar y el compromiso con el aprendizaje continuo.

Este modelo se basa en fortalecer la política educativa: "en integrar en sus procesos los recursos TIC, (...) contar con estándares TIC en educación y su relación con la práctica y la formación docente, facilita el empleo de las TIC, para apoyar los procesos de enseñanza y aprendizaje" (Ascencio et al., 2016, p. 137). Tras el desarrollo del programa se observó su potencial para próximos modelos al apostar por la innovación, no obstante, también mostraron debilidad por las dificultades institucionales acontecidas, ya que no recibieron el apoyo necesario en recursos ni en políticas educativas (Claro \& Jara, 2020).

\subsection{DIGCOMP}

El fomento de la competencia digital en todo Europa era una necesidad, cuando en el 2011, el Institute for Prospective Technological Studies favoreció la creación de un proyecto para crear un marco que sentara las bases para el desarrollo de la competencia digital (Pérez \& Rodríguez, 2016). El mencionado proyecto que estuvo activo entre 2011 y 2013, dio lugar a "DIGCOMP: $A$ Framework for Developing and Understanding Digital Competence in Europe" (Ferrari, 2013). 
Entre algunos de sus objetivos, pretende favorecer el desarrollo de la competencia digital para toda la población, remarca la importancia de la mejora de la formación docente para que sean capaz de responder a las necesidades de sus estudiantes en esta materia. En su desarrollo, se emplearon modelos y enfoques ya existentes para trabajar las diferentes competencias que deben tener todos los ciudadanos y así favorecer la participación plena en la sociedad actual (Alonso-Ferreiro, 2018). Este fue el punto de partida a través del cual, cada estado miembro podría crear sus propias políticas en materia de alfabetización digital (Biel \& Álvarez, 2019). En España el desarrollo de la competencia digital docente fue muy dispar debido a que cada Comunidad Autónoma implementó diferentes políticas (Pérez \& Rodríguez, 2016).

El modelo DigComp instituye cinco áreas competenciales desde las que se articulan veintiuna competencias. Para cada una de las competencias se establecen seis niveles, distribuidos por dificultad y autonomía creciente en: A1 y A2 (Básico), B1 y B2 (Intermedio) y C1 y C2 (Avanzado). Las cinco áreas competenciales recogen las grandes entidades en competencia digital que tendrían que dominar los docentes para poder desarrollar el proceso de enseñanza-aprendizaje correctamente, por medio de una inclusión adecuada de las TIC en el aula (INTEF, 2017):

a) Informatización y alfabetización informacional: navegación, búsqueda y filtrado de información, datos y contenido digital; evaluación de la información, datos y contenidos digitales; almacenamiento y recuperación de información, datos y contenido digital.

b) Comunicación y colaboración: interacción mediante tecnologías digitales; compartir a través de las tecnologías digitales; participación ciudadana en línea; colaboración a través de canales digitales; netiqueta; gestión de la identidad digital.

c) Creación de contenidos digitales: desarrollo de contenidos digitales; integración y reelaboración de contenidos digitales; derechos de autor y licencias; programación.

d) Seguridad: dispositivos y contenido digital, datos personales e identidad; salud y bienestar; entorno.

e) Resolución de problemas: resolución de problemas técnicos; identificación de necesidades y respuestas tecnológica; innovación y uso de la tecnología digital de forma creativa; identificación de lagunas en la competencia digital.

Para poder reconocer el nivel competencial de los docentes en cada una, se establece un descriptor específico que va en consonancia con lo descrito en el Marco para la Competencia Digital del Ciudadano (Carretero et al., 2017).

EI INTEF (2017b) en España, reconoce los niveles competenciales de cada usuario a través del Portfolio de la Competencia Digital Docente. El modelo está basado en un proceso de reflexión sobre el propio nivel de competencia (autoevaluación) y en el registro de evidencias profesionales que demuestre que se han alcanzado los niveles recogidos en las dimensiones.

Entre algunas de las investigaciones que han empleado el modelo DigComp, se encuentran: la de Ibáñez-Etxeberria et al. (2019), enfocada para extrapolar las variables afines con este marco extraídas de 88 apps; Cantón et al. (2017) lo utilizaron para averiguar la dimensión comunicativa del alumnado del grado de Primaria; Jiménez-Hernández et al. (2020) para medir la competencia digital en los de máster o; Pérez \& Rodríguez (2016) en el profesorado de educación Primaria.

En último lugar, se hará una mención especial para DigCompOrg (Comisión Europea, 2016), un modelo desarrollado para facilitar a las organizaciones educativas la integración real y profunda de las tecnologías de aprendizaje digital. A partir de aquí, también puede ser empleada como una herramienta de creación de políticas para la adopción de TIC por las organizaciones educativas, desde niveles regionales hasta europeos.

DigCompOrg genera un marco conceptual integral y genérico adaptable a cada contexto. Además, no pretende reemplazar otros marcos o herramientas ya utilizadas, sino complementarlas. Por eso persigue un doble objetivo, el primero, fomentar la reflexión y evaluación dentro de las organizaciones educativas para mejorar poco a poco el aprendizaje digital y, el segundo, facilitar políticas a cualquier nivel que permitan diseñar y aplicar programas 
para la aplicación de las TIC en el aprendizaje. Para conseguirlo recurre a diferentes elementos vinculados a responsabilidades organizativas (como infraestructura) o individuales (prácticas de enseñanza-aprendizaje; Comisión Europea, 2016).

Un ejemplo de trabajo realizado con este modelo, se encuentra el efectuado por Fernández y Prendes (2020) en Prendes et al. (2021), al utilizarlo para guiar procesos de autodiagnóstico y reflexión sobre la innovación con TIC en organizaciones educativas.

\subsection{DigiLit}

DigiLit Leicester es un proyecto desarrollado por la Universidad de Montfort (2013). Tiene como finalidad dar respaldo a los docentes en el desarrollo de sus conocimientos y destrezas de alfabetización y uso de herramientas digitales, para que posteriormente puedan transferir estos conocimientos al alumnado. Como característica diferenciadora de otros proyectos de evaluación de la competencia digital, utiliza un sistema de evaluación entre pares. DigiLit se compone de seis áreas claves como marco de referencia (Fraser et al., 2013, p. 8):

- Búsqueda, organización y evaluación de la información.

- Creación y compartición de recursos digitales.

- Evaluación y retroalimentación de las prácticas.

- Comunicación, colaboración y participación utilizando las TIC.

- Seguridad electrónica e identidad en línea.

- Desarrollo profesional con soporte tecnológico.

DigiLit ha sido utilizado para la selección de herramientas que faciliten la adquisición de la alfabetización digital, con la precisión del contenido, la calidad para apoyar el desarrollo de la alfabetización, la intuición y la interactividad del usuario, así como textos digitales (Baxa \& Christ, 2018). Silva et al. (2016) lo denominan en base a sus dimensiones como: pionero, desarrollador, básico y para principiantes. Este modelo utiliza estos significados en cada una de las áreas de referencia.

El proyecto DigiLit Leicester en el contexto británico, se especializa en apoyo de las TIC a nivel de profesorado de secundaria, con una perspectiva holística para obtener el desarrollo digital a causa de la falta de conocimientos de los profesionales, por ello se especializa en una autoevaluación así como en trabajo cooperativo para compartir habilidades, saberes y prácticas (Hall et al., 2014).

Para su desarrollo, el proyecto consta de tres etapas (Fraser et al., 2013, p. 5):

- Investigación y búsqueda de literatura sobre educación secundaria y su base empírica.

- Identificar los niveles de confianza y dificultades, en cuanto a la brecha digital en los centros educativos.

- Apoyar al personal docente en el desarrollo de sus habilidades y confianza desde su práctica efectiva y con carácter innovador.

Este modelo permite adoptar un enfoque cooperativo y asociativo para la innovación pedagógica. Además, apoya que las cuestiones sociales y éticas respalden un enfoque en la agencia docente y la colegialidad dentro del dominio de la alfabetización digital (Hall et al., 2014). Por ello, se han basado en Personal Learning Networks (PLN) para reforzar el aprendizaje digital a través de discutir y compartir ideas, recursos y enfoques de interés a partir de las prácticas de los propios educadores (Fraser et al., 2013).

\subsection{COMDID}

Este modelo parte de un proyecto realizado por la Universidad Roviri i Virgili (2014-2016) denominado "Estrategia formativa para el desarrollo de la competencia digital docente", con el objetivo principal de diseñar y elaborar unos instrumentos para la evaluación de conocimientos vinculados a la CDD, acercando lo teórico a lo práctico en la doble titulación de Grado (Educación Infantil y Educación Primaria). 
Para el modelo COMDID, Lázaro \& Gisbert (2015a, 2015b) han publicado diferentes instrumentos/rúbricas de evaluación en cuatro dimensiones: didáctica, curricular y metodológica; planificación, organización y gestión de espacios y recursos; relación ética y seguridad; y personal y profesional. Los escenarios son: el aula, el centro educativo, la comunidad educativa y el entorno; y el desarrollo profesional: principiante, medio, experto y transformador.

Existe una relación directa entre el nivel de desarrollo de la competencia digital del profesorado y el uso didáctico que se da en el aula (Lázaro et al., 2018). Por tanto, la formación inicial docente tendría que incluir un currículum digital inclusivo de manera que todos dispongan de una formación similar en el ámbito TIC y de herramientas que favorezcan el compartimento de experiencias de calidad y el reciclaje. Lazaro \& Gisbert (2015b) apuntan a la necesidad de realizar esta formación desde los primeros cursos de grado y a la vez, sea revisado a nivel interuniversitario y regido por las instituciones responsables de la acreditación, tanto del profesorado como de los contenidos.

Como nueva sugerencia de estos instrumentos de evaluación, los autores Lázaro et al. (2019), avanzarían en una retroalimentación rápida y sobre cómo mejorarían habilidades para la enseñanza y aprendizaje. Autores como García et al. (2015), señalan la importancia en este modelo de percibir el grado de desarrollo de la CDD desde la experiencia, conocimiento y capacidad, en los diferentes contextos educativos en relación también a su propio desarrollo personal y profesional.

\subsection{Comparación de los modelos}

Finalmente, para establecer una mejor comparativa entre los diferentes modelos de CDD estudiados, se presenta una tabla resumen con las principales características de cada uno de ellos (tabla 3).

Tabla 3. Resumen de los modelos de CDD

\begin{tabular}{|c|c|c|c|c|c|}
\hline & $\begin{array}{c}\text { Institución y } \\
\text { año }\end{array}$ & Población & $\begin{array}{l}\text { Características } \\
\text { o dimensiones }\end{array}$ & Fines & $\begin{array}{l}\text { Investig } \\
\text { aciones }\end{array}$ \\
\hline ECD-TIC & UNESCO (2008) & $\begin{array}{l}\text { Profesorado } \\
\text { en formación y } \\
\text { ejerciente }\end{array}$ & $\begin{array}{l}\text { Alfabetización } \\
\text { tecnológica, } \\
\text { profundización } \\
\text { y creación de } \\
\text { conocimientos. }\end{array}$ & $\begin{array}{l}\text { Integrar las } \\
\text { TIC en la } \\
\text { docencia, } \\
\text { formación del } \\
\text { profesorado y } \\
\text { sistemas } \\
\text { escolares. }\end{array}$ & $\begin{array}{l}\text { Valdivies } \\
\text { o \& } \\
\text { Gonzále } \\
\text { s (2016) }\end{array}$ \\
\hline NETS-T & ISTE (2008) & $\begin{array}{l}\text { Profesorado } \\
\text { ejerciente }\end{array}$ & $\begin{array}{c}\text { Facilitar el } \\
\text { aprendizaje, } \\
\text { diseño de } \\
\text { prácticas, } \\
\text { herramientas } \\
\text { digitales, e- } \\
\text { ciudadano y } \\
\text { crecimiento } \\
\text { profesional. }\end{array}$ & $\begin{array}{c}\text { Ayudar a la } \\
\text { implantación } \\
\text { de las TIC en } \\
\text { el aula, } \\
\text { formación } \\
\text { docente y } \\
\text { ciudadanía } \\
\text { digital. }\end{array}$ & $\begin{array}{c}\text { Banister } \\
\quad \& \\
\text { Vannatta } \\
\text { (2012); } \\
\text { Esteve } \\
\text { et al. } \\
\text { (2016a y } \\
\text { 2016b); } \\
\text { Kumar \& } \\
\text { Vigil } \\
\text { (2014) }\end{array}$ \\
\hline ISTE II & ISTE (2017) & $\begin{array}{l}\text { Profesorado y } \\
\text { alumnado }\end{array}$ & $\begin{array}{c}\text { Formación, uso } \\
\text { de TIC, } \\
\text { incentivar } \\
\text { aprendizaje, } \\
\text { diseño y } \\
\text { análisis de } \\
\text { aprendizaje. }\end{array}$ & $\begin{array}{c}\text { Capacitación } \\
\text { digital docente, } \\
\text { actualización, } \\
\text { buenas } \\
\text { prácticas y } \\
\text { autonomía } \\
\text { estudiantil. }\end{array}$ & $\begin{array}{l}\text { Biel \& } \\
\text { Álvarez } \\
\text { (2019); } \\
\text { Torrey } \\
\text { (2018). }\end{array}$ \\
\hline
\end{tabular}




\begin{tabular}{|c|c|c|c|c|c|}
\hline ENLACES & $\begin{array}{l}\text { Ministerio de } \\
\text { Educación de } \\
\text { Chile (2006) }\end{array}$ & $\begin{array}{c}\text { Profesorado } \\
\text { ejerciente }\end{array}$ & $\begin{array}{l}\text { Pedagógica, } \\
\text { técnica, } \\
\text { gestión, social y } \\
\text { responsabilidad } \\
\text { profesional. }\end{array}$ & $\begin{array}{c}\text { Definir } \\
\text { competencia } \\
\text { en TIC de los } \\
\text { docente y } \\
\text { programas de } \\
\text { formaciones. }\end{array}$ & $\begin{array}{c}\text { Ascenci } \\
\text { o et al } \\
(2016) ; \\
\text { Claro \& } \\
\text { Jara } \\
(2020)\end{array}$ \\
\hline DIGCOMP & $\begin{array}{c}\text { Comisión } \\
\text { Europea } \\
\text { (Ferrari, 2013) }\end{array}$ & $\begin{array}{c}\text { Profesorado y } \\
\text { comunidad } \\
\text { educativa }\end{array}$ & $\begin{array}{c}\text { Desarrollo de la } \\
\text { competencia } \\
\text { digital en la } \\
\text { población y } \\
\text { mejora de la } \\
\text { formación } \\
\text { docente. }\end{array}$ & $\begin{array}{l}\text { Alfabetización } \\
\text { informacional, } \\
\text { comunicación, } \\
\text { contenidos } \\
\text { digitales, } \\
\text { seguridad y } \\
\text { resolución de } \\
\text { problemas. }\end{array}$ & $\begin{array}{c}\text { Ibáñez- } \\
\text { Etxeberri } \\
\text { a et al. } \\
\text { (2019); } \\
\text { Cantón } \\
\text { et al. } \\
\text { (2017); } \\
\text { Jiménez } \\
\text { - } \\
\text { Hernánd } \\
\text { ez et al. } \\
\text { (2020), } \\
\text { entre } \\
\text { otras }\end{array}$ \\
\hline DigiLit & $\begin{array}{l}\text { Universidad de } \\
\text { Montfort (2013) }\end{array}$ & $\begin{array}{c}\text { Profesorado } \\
\text { ejerciente en } \\
\text { especial de } \\
\text { secundaria }\end{array}$ & $\begin{array}{c}\text { Desarrollar } \\
\text { destrezas, } \\
\text { conocimientos y } \\
\text { uso de } \\
\text { herramientas } \\
\text { TIC. }\end{array}$ & $\begin{array}{l}\text { Búsqueda de } \\
\text { información, } \\
\text { comunicación } \\
\text { TIC, creación } \\
\text { de recursos, } \\
\text { soporte } \\
\text { tecnológico, } \\
\text { evaluación y } \\
\text { seguridad. }\end{array}$ & $\begin{array}{c}\text { Baxa \& } \\
\text { Christ } \\
(2018)\end{array}$ \\
\hline COMDID & $\begin{array}{l}\text { Universidad } \\
\text { Roviri i Virgili } \\
(2014-2016)\end{array}$ & $\begin{array}{l}\text { Profesorado } \\
\text { en formación y } \\
\text { ejerciente }\end{array}$ & $\begin{array}{c}\text { Diseñar } \\
\text { instrumentos de } \\
\text { evaluación } \\
\text { teórico- } \\
\text { prácticos y de } \\
\text { conocimientos } \\
\text { de CDD. }\end{array}$ & $\begin{array}{l}\text { Didáctica, } \\
\text { gestión de } \\
\text { espacios, ética } \\
\text { y personal. }\end{array}$ & $\begin{array}{l}\text { Lázaro } \\
\text { et al. } \\
\text { (2019) }\end{array}$ \\
\hline
\end{tabular}

\section{DISCUSIÓN Y CONCLUSIONES}

Llegados a este punto, con este estudio se ha pretendido retratar la situación actual en materia de CDD. El fin que cada uno de los modelos persigue, no es otro que la mejora de la calidad educativa de cada país o región, a través del favorecimiento de políticas educativas y formaciones docentes, tanto inicial como permanente, para adquirir estas competencias.

Los modelos, mediante sus dimensiones y fines buscan repercutir en los estándares de formación del profesorado, para que sean adaptados a las apremiantes necesidades sociales del momento y se llegue a una universalización de la competencia digital en la población. En consonancia a lo descrito, para Lugo \& Ruiz (2016) estos modelos han sido considerados prioridades educativas de cada país, con una mirada convergente que formule fácilmente una educación de calidad e inclusiva, y donde las políticas públicas faciliten la democratización del conocimiento. 
A partir de su revisión, se ha podido observar como todos los modelos siguen una misma lógica interna en base a la formación TIC del profesorado que repercuta en su docencia. No obstante, todos los modelos han mostrado discrepancias entre sí. Por ejemplo, menos DigiLit y COMDID que se centralizan en docentes ejercientes o en formación, el resto, en mayor o menor medida, tiene por objetivo el desarrollo digital y participación social de los ciudadanos, en el caso de NEST-T, hace un especial énfasis al denominarlos como e-ciudadanos, a aquellos que son digitalmente responsables y realizan un uso ético de las herramientas. Otro aspecto que puede diferenciarlos, es su lugar de actuación, así, DigComp nació para asentar las bases digitales de los países europeos, otros como NEST-T o Enlaces actúan a nivel nacional, en este caso, EE. UU y Chile. Asimismo, hay modelos que se crean en una institución concreta, como DigiLit en la Universidad de Montfort o COMDID en la Universidad Roviri i Virgili. Finalmente, también habría que remarcar que el tratamiento diferencia de la innovación pedagógica y, aunque en todos está vinculado, solo se trata de manera más central y explícita en ECD-TIC y DigiLit.

A través de las diferentes investigaciones presentadas en el artículo (Banister \& Vannatta, 2012; Ballesta et al., 2016; Jiménez-Hernández et al., 2020; Pérez \& Rodríguez, 2016; entre otras), se ha podido corroborar que toda la información expuesta no se queda en mera teoría, sino que hay muchas vías de trabajo en materia digital hechas, y más que se continúan haciendo.

No obstante, existen puntos negativos que han quedado evidenciados y que son importantes analizar, por ejemplo, Biel \& Álvarez (2019), reconocen la falta de formación en competencia digital en docentes del Espacio Europeo de Educación Superior. Como se ha dicho anteriormente, es un asunto clave y fundamental para poder hacer que todos los estudiantes dispongan de esta competencia. Además, tras el estudio realizado por Esteve et al. (2018) sobre los proyectos de CDD, observan que todas las investigaciones tienen la misma carencia, y es considerar al profesorado únicamente en su función en el aula, obviando el compromiso con la comunidad social y educativa, además de mantener una reducción taxonómica de la labor docente sin cabida en aspectos más holísticos, y por último, mantener una visión contradictoria en el determinismo entre sociedad y tecnología. Esteve et al. (2018) abren una nueva concepción en cuanto a la necesidad de ver cada contexto, así como sus capacidades, como necesarias para adquirir la competencia docente integral en el mundo digital.

En último lugar además de la función formadora, también se ha podido verificar que, de una manera $u$ otra, en todos los sistemas educativos se desarrollan y profundizan en modelos de CDD, buscando implementar de manera estandarizada competencias TIC en las aulas, aplicando de manera didáctica diferentes recursos y metodologías. A la vez, pervive una necesidad de hacer visible a ojos de instituciones educativas, un cuidado en las capacidades adquiridas y por adquirir, tanto en docentes como en políticas institucionalizadas. Y queda patente que, aunque queda camino por recorrer, es cierto que se está avanzando en el desarrollo digital del alumnado y del profesorado y, por lo tanto, los frutos de estas mejoras poco a poco se irán experimentando en diferentes momentos y situaciones como los nuevos retos que se están produciendo en momentos actuales con la formación online, que, forzada o elegida, está haciendo evolucionar estos modelos de CDD.

\section{REFERENCIAS BIBLIOGRÁFICAS}

Aguirre, J.C., \& Jaramillo, L.G. (2015). El papel de la descripción en la investigación cualitativa. Cinta de moebio, 53, 175-189. http://dx.doi.org/10.4067/S0717-554X2015000200006

Alonso-Ferreiro, A. (2018). Aprendizaje basado en proyectos para el desarrollo de la competencia digital docente en la formación inicial del profesorado. Relatec: Revista Latinoamericana de Tecnología Educativa, 17(1), 9-24. https://bit.ly/3cWfYYD

Ascencio, P.B., Garay, M.F., \& Seguic, E. (2016). Formación inicial docente (FID) y tecnologías de la información y comunicación (TIC) en la Universidad de Magallanes-Patagonia Chilena. Digital Education Review, 30, 123-134. https://bit.ly/3ta9WJT

Ballesta, J., Martínez, J., \& Céspedes, R. (2017). Un modelo integrador para la alfabetización mediática y la competencia digital en Educación Primaria. Revista Fuentes, 19(2), 139-154. https://bit.ly/3cSK7lq 
Banister, S., \& Vannata, R.V. (2014). Assessing NETS - T Performance in Teacher Candidates. Journal of Digital Learning in Teacher Education, 29(2), 59-65. https://doi.org/10.1080/21532974.2012.10784705

Baxa, J., \& Christ, T. (2018). The DigiLit framework. The Reading Teacher, 71(6), 703-714. https://doi.org/10.1002/trtr.1660

Biel, L.A., \& Álvarez, E. (2019). La competencia digital docente del profesor universitario 3.0. Caracteres: estudios culturales y críticos de la esfera digital, 8(2), 205-236. https://bit.ly/3uvf8Il

Bilbao, A., \& Salinas, A. (2010). El libro abierto de la informática educativa. Enlaces. https://bit.ly/3fPWqHw

Cantón, I., Cañón, R., \& Grande, M. (2017). La comunicación como subdimensión de la competencia digital en futuros maestro de primaria. Píxel-Bit. Revista de Medios y Educación, 50, 33-47. http://dx.doi.org/10.12795/pixelbit.2017.i50.02

Carretero, S., Vuorikari, R., \& Punie, Y. (2017). DigComp 2.1. The Digital Competence Framework for Citizens. European Commision. https://bit.ly/31W9seh

Castañeda, L., Esteve, F., \& Adell, J. (2018). ¿Por qué es necesario repensar la competencia docente para el mundo digital?. Revista de Educación a Distancia (RED), 56. http://dx.doi.org/10.6018/red/56/6

Claro, M., \& Jara, I. (2020). The end of Enlaces: 25 years of an ICT education policy in Chile. Digital Education Review, 37, 96-108. https://doi.org/10.1344/der.2020.37.96-108

Comisión Europea (2016). DigCompOrg. Digitally Competent Educational Organisations. https://bit.ly/3cXruDm

Durán, M., Prendes, M.P., \& Guriérrez, I. (2019). Certificación de la Competencia Digital Docente: propuesta para el profesorado universitario. RIED. Revista lberoamericana de Educación a Distancia, 22(1), 187-205. http://dx.doi.org/10.5944/ried.22.1.22069

Enlaces. (2011). Competencias y Estándares TIC para la Profesión Docente. https://bit.ly/3sX5v4T

Esteve, F.M. (2015). La competencia digital docente: análisis de la autopercepción y evaluación del desempeño de los estudiantes universitarios de educación por medio de un entorno $3 D$ [Tesis Doctoral, Universitat Rovira i Virgili]. https://bit.ly/3fQusLA

Esteve-Mon, F.M., Castañeda, L., \& Adell-Segura, J. (2018). Un modelo holístico de competencia docente para el mundo digital. Revista Interuniversitaria de Formación del Profesorado, 91, 105-116. https://bit.ly/3uvwvZK

Esteve, F.M., Gisbert, M., \& Lázaro, J.L. (2016a). La Competencia Digital de los futuros docentes: ¿Cómo se ven los actuales estudiantes de educación?. Perspectiva Educacional, Formación de Profesores, 55(2), 38-54. https://bit.ly/3usIYOO

Esteve, F., Cela, J.M., \& Gisbert, M. (2016b). ETeach3D: Designing a 3D Virtual Environment for Evaluating the Digital Competence of Preservice Teachers. Journal of Educational Computing Research, 54(6), 816-839. https://doi.org/10.1177\%2F0735633116637191

Falcó, J.M. (2017). Evaluación de la competencia digital docente en la comunidad autónoma de Aragón. Revista Electrónica de Investigación Educativa, 19(4), 73-83. https://doi.org/10.24320/redie.2017.19.4.1359

Ferrari, A. (2013). DIGCOMP: A framework for developing and understanding digital competence in Europe. IPTS. European Comission. https://bit.ly/3s5Injz

Fraser, J., Atkins, L., \& Hall, R. (2013). DigiLit Leicester. Supporting teachers, promoting digital literacy, transforming learning. Leicester City Council. https://bit.ly/3wv0UJv

Garcia i Grau, F., Lázaro-Cantabrana, J.L., \& Valls-Bautista, C. (2020). Avanzando hacia la madurez digital del centro educativo: un análisis de la competencia digital docente. En E. Sánchez, E. Colomo, J. Ruiz y J. Sánchez (Eds.), Tecnologías educativas y estrategias didácticas (pp. 188-199). Universidad de Málaga. https://bit.ly/2PzK2Al 
Girón, V., Cózar, R., \& González, J.A. (2019). Análisis de la autopercepción sobre el nivel de competencia digital docente en la formación inicial de maestros/as. Revista Electrónica Interuniversitaria de Formación del Profesorado, 22(3), 193-218. https://doi.org/10.6018/reifop.373421

Gisbert, M., \& Esteve, F. (2016). Digital Leaners: la competencia digital de los estudiantes universitarios. La cuestión universitaria, 7, 48-59. https://bit.ly/39NtM5G

Guillén-Gámez, F.D., Álvarez-García, F.J., \& Rodríguez, I.M. (2018). Digital tablets in the music classroom: A study about the academic performance of students in the BYOD context. Journal of Music, Technology \& Education, 11(2), 171-182. https://doi.org/10.1386/jmte.11.2.171 1

Hall, R., Atkins, L., \& Fraser, J. (2014). Defining a self-evaluation digital literacy framework for secondary educators: the DigiLit Leicester project. Research in Learning Technology, 22. https://doi.org/10.3402/rlt.v22.21440

Ibáñez-Etxeberria, A., Kortabitarte, A., De Castro, P., \& Gillate, I. (2019). Competencia digital mediante apps de temática patrimonial en el marco DigComp. Revista Electrónica Interuniversitaria de Formación del Profesorado, 22(1). http://dx.doi.org/10.6018/reifop.22.1.356231

INTEF (2017a). Marco Común de Competencia Digital Docente. Ministerio de Educación Cultura y Deporte. https://bit.ly/2RdzMOW

INTEF (2017b). Portfolio de la Competencia Digital Docente. Ministerio de Educación Cultura y Deporte. https://bit.ly/39NvF2g

ISTE (2008). National Educational Technology Standards for Teachers. International Society for Technology in Education.

ISTE. (2017). ISTE Standards for Educators. International Society for Technology in Education.

Jiménez-Hernández, D., González-Calatayud, V., Torres-Soto, A., Martínez, A. \& Morales, J. (2020). Digital Competence of Future Secondary School Teachers: Differences According to Gender, Age, and Branch of Knowledge. Sustainability, 12(22), 9473. https://doi.org/10.3390/su12229473

Kitchenham, B. (2004). Procedures for performing systematic reviews. Keele, UK, Keele University, 33, 1-26. https://bit.ly/3fLqvYF

Krumsvik, R. (2011). Digital competence in Norwegian teacher education and schools. Högre utbildning, 1(1), 39-51. https://bit.ly/3uv3ayt

Kumar, S., \& Vigil, K. (2014). The Net Generation as Preservice Teachers. Journal of Digital Learning in Teacher Education, 27(4), 144-153. https://doi.org/10.1080/21532974.2011.10784671

Lázaro, J.L. \& Gisbert, M.G. (2015a). El desarrollo de la competencia digital docente a partir de una experiencia piloto de formación en alternancia en el Grado de Educación. Educar, 51(2), 321-348. https://doi.org/10.5565/rev/educar.725

Lázaro, J.L., \& Gisbert, M.G. (2015b). Elaboració d'una rúbrica per avaluar la competència digital del docent. Universitas Tarraconensis. Revista de Ciències de l'Educació, 1(1), 48-63. https://doi.org/10.17345/ute.2015.1.648

Lázaro, J.L., Usart, M., \& Gisbert, M. (2019). Assessing Teacher Digital Competence: the Construction of an Instrument for Measuring the Knowledge of Pre-Service Teachers. Journal of New Approaches in Educational Research, 8(1), 73-78. https://doi.org/10.7821/naer.2019.1.370

Ley Orgánica 8/2013, de 9 de diciembre, para la Mejora de la Calidad Educativa (LOMCE), Boletín Oficial del Estado (BOE) 2013, de 10 de diciembre, 1-64. https://bit.ly/3mp6QPz

Morphew, V.N. (2012). A Constructivist Approach to the National Educational Technology Standards for Teachers. International Society for Technology in Education (ISTE). 
Muñoz, P. (2016). Estrategias descolonizadoras en competencias culturales para la formación de educadores. Opción, 32(12), 66-84. https://bit.ly/3ut0XUb

Pérez, A., \& Rodríguez, M.J. (2016). Evaluación de las competencias digitales autopercibidas del profesorado de educación primaria en Castilla y León. Revista de Investigación Educativa, 34 (2), 399-415. http://doi.org/10.6018/rie.34.2.215121

Pike, K.L. (1967). Etic and emic standpoints for the description of behavior. En K.L. Pike, Language in relation to a unified theory of the structure of human behavior (pp. 37-72). Mouton \& Co. https://doi.org/10.1037/14786-002

Prendes, M.P., Román, M., \& González-Calatayud, V. (2021). Modelo integral de análisis de la competencia digital en las instituciones de enseñanza superior: profesorado y estudiantes en el contexto organizativo. Revista Panorâmica, 32. https://bit.ly/3dzCO7q

Resolución de 2 de julio de 2020, de la Dirección General de Evaluación y Cooperación Territorial, por la que se publica el Acuerdo de la Conferencia Sectorial de Educación sobre el marco de referencia de la competencia digital docente. Boletín Oficial del Estado, 191, sec. III, de 13 de julio de 2020, 50639-50668. https://bit.ly/3t1dMog

Rodrigo. M. (2001). Teorías de la comunicación: ámbitos, métodos y perspectivas. Bellaterra.

Silva, J., Miranda, P., Gisbert, M., Morales, M., \& Onetto, A. (2016). Indicadores para evaluar la competencia digital docente en la formación inicial en el contexto Chileno - Uruguayo. RELATEC: Revista Latinoamericana de Tecnología Educativa, 15(3), 55-67. http://dx.doi.org/10.17398/1695-288X.15.3.55

Torrey, Trust. (2018). ISTE Standards for Educators: From Teaching With Technology to Using Technology to Empower Learners. Journal of Digital Learning in Teacher Education, 34(1), 13. https://doi.org/10.1080/21532974.2017.1398980

UNESCO (2008). Estándares de competencias en TIC para docentes. https://bit.ly/31Uaj16

UNESCO (2011). UNESCO ICT competency framework for teachers. United Nations Educational, Scientific and Cultural Organization (UNESCO).

Valdivieso, T.S., \& Gonzáles, M.Á. (2016). Competencia digital docente: ¿Dónde estamos?. Perfil del docente de educación primaria y secundaria. El caso de Ecuador. Pixel-Bit: Revista de Medios y Educación, 49, 57-73. https://bit.ly/3wBwV2w

\section{INFORMACIÓN SOBRE LOS AUTORES}

\section{David Jiménez-Hernández}

Universidad Miguel Hernández

David Jiménez-Hernández es Licenciado en Pedagogía y con Máster en Investigación e Innovación en Educación Infantil y Primaria por la Universidad de Murcia. Doctor en Ciencias Sociales y de la Comunicación por la Universidad Católica San Antonio de Murcia, donde estuvo como docente e investigador durante seis años, adscrito a los grados de Educación Infantil y Primaria. Actualmente es Ayudante Doctor en la Universidad Miguel Hernández de Elche, impartiendo su docencia en el Máster de Formación del Profesorado. Sus principales líneas de investigación, se han dirigido al estudio de métodos didácticos, la formación del profesorado y las Tecnologías de la Información y la Comunicación.

ResearchGate: https://www.researchgate.net/profile/David-Jimenez-39

\section{Práxedes Muños Sánchez}

Universidad Católica de Murcia 
Práxedes Muñoz Sánchez es Dra. en Antropología Social y Cultural, Profesora e investigadora en la Universidad Católica de Murcia, España, en el Grado de Educación Primaria, Grado de Turismo, Máster de Desarrollo Social y Máster de Formación del Profesorado desde 2011. Estancias de investigación en Guatemala, México, Cuba y Chile. Docente de cursos de Doctorado y Maestrías en universidades de Chile y México. Líneas de investigación: Antropología de la Educación, Investigación Acción, Antropología del Desarrollo, movimientos sociales, género, violencia de género, competencia cultural, (de)colonización, cooperación al desarrollo y patrimonio. Artículos publicados en bases de datos en Scimago y Latindex; capítulos de libro y monografías. Proyectos de investigación en innovación docente y proyectos europeos sobre Ninis, Violencia de Género y Patrimonio de la memoria.

\section{Fulgencio S. Sánchez Giménez}

IES Jaume Primer Ontinyent

Fulgencio S. Sánchez Giménez es profesor de Formación Profesional en activo en el instituto IES Jaume I de Ontinyent (Consellería d'Educació Generalitat Valenciana). Imparte docencia en Ciclos Formativos relacionados con la familia de Sanidad. Ha realizado el grado en Magisterio, Mención en TIC. Ha concluido, entre otros, el Máster Universitario e-Learning y Tecnología Educativa en Educación (Universidad Camilo José Cela), cosa que le ha permitido ser coordinador de Seminarios y Grupos de Trabajo y ponente de varios cursos para el Centro de Formación del Profesorado de la Generalitat Valenciana, relacionados con la implementación de las TIC en el aula y en el manejo de éstas. Ha escrito varios libros, entre los que destaca "Competencias Digitales para el Profesorado de la Comunidad Valenciana". Es autor de diversas publicaciones en revistas internacionales relacionadas con la Innovación Educativa y la medición del logro de los estándares digitales en educación.

Los textos publicados en esta revista están sujetos a una licencia de Reconocimiento 4.0 España de Creative Commons. Puede copiarlos, distribuirlos, comunicarlos públicamente y hacer obras derivadas siempre que reconozca los créditos de las obras (autoría, nombre de la revista, institución editora) de la manera especificada por los autores o por la revista. La licencia completa se puede consultar en:Licencia Creative Commons Atribución-NoComercial-Compartir por igual 4.0 Internacional. 